\title{
Meningeal carcinomatosis from bladder cancer: A case report and review of the literature
}

\author{
YUTA UMEZAWA $^{1}$, SUGURU SHIROTAKE ${ }^{1}$, GO KANEKO $^{1}$, KOSHIRO NISHIMOTO ${ }^{1}$, YOSHITAKA OKADA ${ }^{2}$, \\ AKIRA UCHINO $^{2}$, MASANORI YASUDA ${ }^{3}$ and MASAFUMI OYAMA ${ }^{1}$
}

Departments of ${ }^{1}$ Uro-Oncology, ${ }^{2}$ Diagnostic Radiology and ${ }^{3}$ Diagnostic Pathology, Saitama Medical University International Medical Center, Hidaka, Saitama 350-1298, Japan

Received October 3, 2018; Accepted February 4, 2019

DOI: $10.3892 / \mathrm{mco} .2019 .1820$

\begin{abstract}
A 66-year-old Japanese male patient was referred to Saitama Medical University International Medical Center for treatment of bladder cancer (clinical stage T2 or higher without metastasis), and underwent radical cystectomy with pelvic lymphadenectomy. The histopathological diagnosis was high-grade urothelial carcinoma (pathological stage T2bN2, ly1, v0) and 2 cycles of adjuvant systemic chemotherapy (gemcitabine plus cisplatin) were administered. At 15 months after the operation, mediastinal and lung hilar lymph nodes and multiple bone metastases were identified on computed tomography imaging. After 3 cycles of the previous regimen as salvage systemic chemotherapy, the lymph node metastases had shrunk and the bone metastases were stable; therefore, further chemotherapy was planned. At 26 days after the initiation of the 4th cycle, the patient felt nausea and lower limb weakness. Spinal and brain magnetic resonance imaging with contrast medium revealed diffuse enhancement at the surface of the spinal cord and brain. In addition, abnormal signal intensity in the subarachnoid space was observed on fluid-attenuated inversion recovery imaging; therefore, the patient was diagnosed with meningeal carcinomatosis (MC). Treatment, including whole-brain radiotherapy, was planned for MC; however, the patient's condition rapidly worsened and he succumbed to the disease 14 days after the diagnosis of MC. The definitive diagnosis of MC was confirmed at autopsy.
\end{abstract}

\section{Introduction}

Meningeal carcinomatosis (MC) is defined as the spread of tumor cells within the leptomeninges and the subarachnoid space (1). MC is diagnosed in 1-5\% of patients with solid tumors, $5-15 \%$ of patients with leukemia and lymphoma, and

Correspondence to: Dr Suguru Shirotake, Department of Uro-Oncology, Saitama Medical University International Medical Center, 1397-1 Yamane, Hidaka, Saitama 350-1298, Japan

E-mail: ss197816@saitama-med.ac.jp

Key words: meningeal carcinomatosis bladder, cancer autopsy
$1-2 \%$ of patients with primary brain tumors (2). Among solid tumors, breast cancer (12-35\%), lung cancer (10-26\%) and melanoma $(5-25 \%)$ represent the most common causative cancers resulting in $\mathrm{MC}$ (2-6). The prognosis from initial diagnosis as $\mathrm{MC}$ in solid tumors is $\sim 6-8$ weeks. MC-directed treatment may contribute to improved survival of a few months (1). We herein present a rare case of MC from bladder cancer with lymph node and bone metastases. The case is reviewed based on pathological examination at autopsy.

\section{Case report}

A 66-year-old Japanese male patient visited a local hospital with complaints of asymptomatic gross hematuria in January 2014. The patient had a medical history of diabetes mellitus and a smoking history of $>40$ years (unspecified smoking amount). The urine cytological examination detected urothelial cancer cells; however, the patient refused further examination or treatment at that time. In April 2015, the patient experienced pain on urination and visited the hospital again. A computed tomography (CT) scan revealed a thickened anterior bladder wall, which was suspicious for bladder cancer; no metastases were identified. Following transurethral resection of the bladder tumor, the pathological diagnosis was high-grade muscle-invasive urothelial carcinoma and the patient was referred to the Saitama Medical University International Medical Center (Hidaka, Japan) to receive radical treatment in June 2015 (SIMC-Uro \#8526: a unique non-sequential patient control number in the Department of Uro-Oncology, Saitama Medical University International Medical Center). No obvious abnormalities were found on physical examination during the first visit. Laboratory examination revealed slight elevation of the serum C-reactive protein levels $(0.444 \mathrm{mg} / \mathrm{dl}$, normal range: $0.000-0.250 \mathrm{mg} / \mathrm{dl}$ ) and positive for urothelial cancer cells in urine cytology, without any abnormal findings in particular. The patient declined neoadjuvant chemotherapy, and radical cystectomy with pelvic lymphadenectomy was performed. The pathological diagnosis was high-grade urothelial carcinoma, pT2b (Fig. 1A) N2 (2 of 7 pelvic lymph nodes), and lymphatic vessel invasion (Fig. 1B). Starting at 2 months after radical cystectomy, two cycles of adjuvant systemic chemotherapy with gemcitabine $\left(1,000 \mathrm{mg} / \mathrm{m}^{2}\right.$ on days 1,8 and 15$)$ and cisplatin (70 mg/m $\mathrm{m}^{2}$ on day 2 ) (GC chemotherapy) 
A

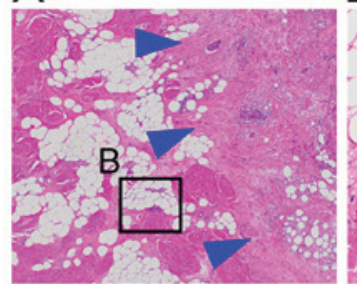

E

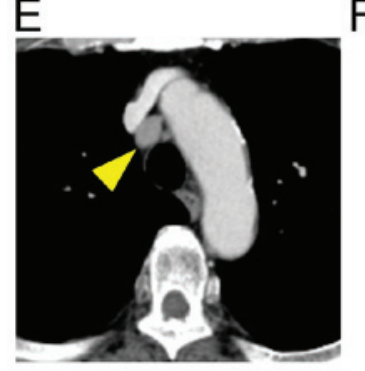

B

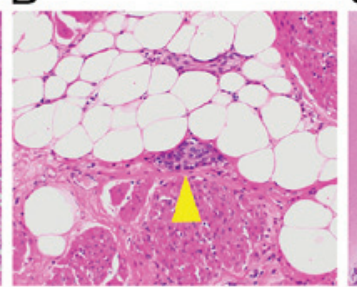

F

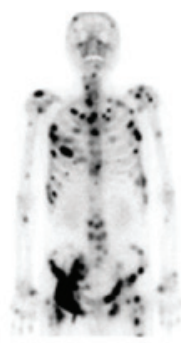

C

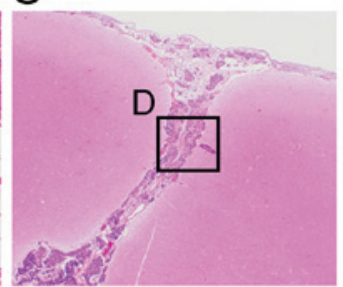

G

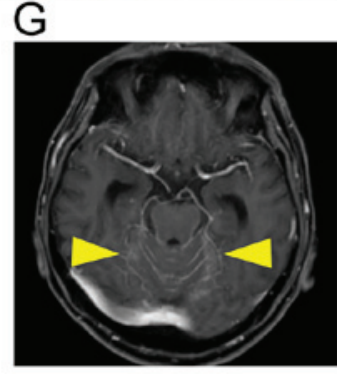

D

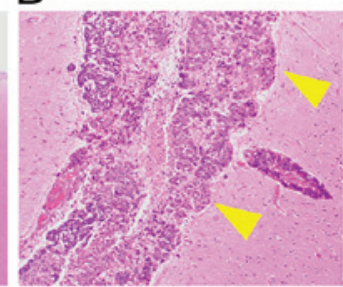

$\mathrm{H}$

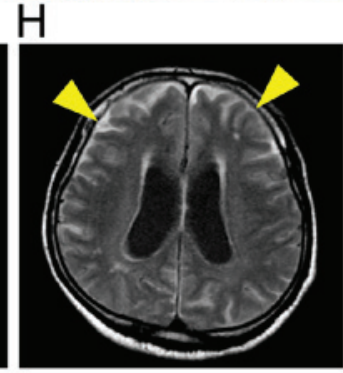

I

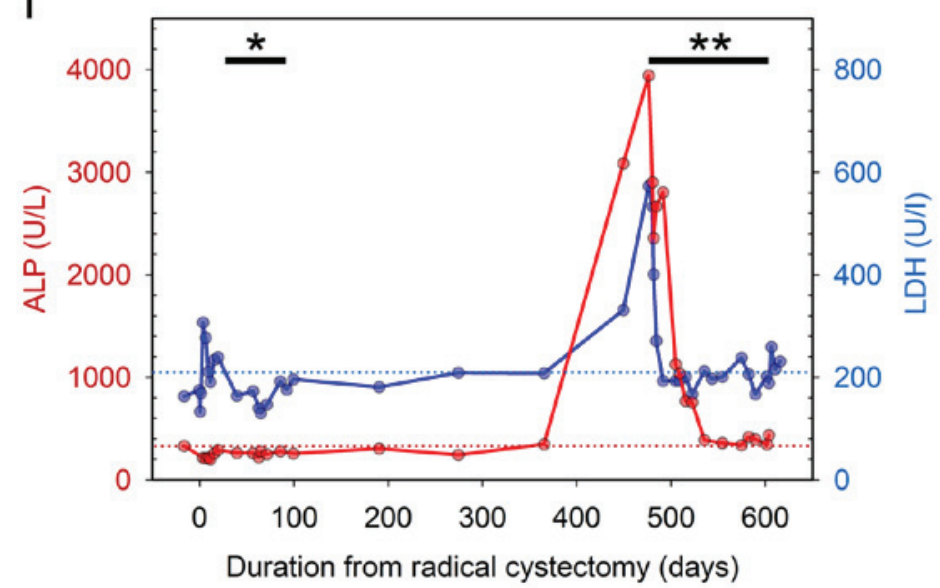

Figure 1. Pathological and radiographic images, and the change of laboratory data during the clinical course. (A and B) Histopathological images of the bladder following radical cystectomy (A, magnification, x20; B, x400 magnification of the square in A). Tumor cells infiltrated the bladder muscle layer (blue arrowheads in A), and invaded lymphatic vessels (yellow arrowhead in B). (C and D) Pathological images of brain at autopsy (C, magnification, x20; D, x400 magnification of the square in C). Tumor cells infiltrated the subarachnoid space and cerebral sulcus (arrowheads). (E) Chest computed tomography images with enhancement showing lymph node metastasis in the mediastinum (arrowhead). (F) Bone scan image at 15 months after cystectomy showing multiple bone metastases. ( $\mathrm{G}$ and $\mathrm{H}$ ) Brain magnetic resonance imaging at diagnosis of meningeal carcinomatosis (G, post-contrast T1-weighted image; H, FLAIR image). The arrowheads indicate $(\mathrm{G})$ abnormal enhancement of the brainstem surface and cerebellar fissure and $(\mathrm{H})$ diffuse abnormal high signal intensity in the subarachnoid space; hydrocephalus is also observed. (I) Change in laboratory data during the clinical course of the disease. Red and blue lines show changes in serum ALP and LDH levels, respectively. Red and blue dotted lines show the upper limit of normal for serum ALP (338 U/l) and LDH levels (211 U/l), respectively. ", Duration of adjuvant chemotherapy; ${ }^{* *}$, duration of salvage chemotherapy. ALP, alkaline phosphatase; LDH, lactate dehydrogenase.

were administered. Follow-up consisted of physical examination, routine blood work, and chest and abdominal CT imaging every 3 months. At 15 months after radical cystectomy, despite the lack of symptoms, CT imaging revealed multiple enlarged lymph nodes [including mediastinal (Fig. 1E) and lung hilar nodes] and osteosclerotic changes in multiple bones, while a bone scan revealed multiple systemic hot spots (Fig. 1F). In addition, there was a sudden increase in serum alkaline phosphatase (ALP; 3,943 U/1, normal range: 104-338 U/1) and lactate dehydrogenase $(\mathrm{LDH} ; 573 \mathrm{U} / 1$, normal range: 106-211 U/l) levels compared with previous data (Fig. 1I). For multiple lymph node and bone metastases following radical cystectomy with adjuvant chemotherapy, salvage GC chemotherapy (at the same dosage as previous adjuvant GC chemotherapy) and denosumab (120 mg, once monthly) were administered. CT imaging and bone scan after 3 months of GC chemotherapy revealed that lymph node and bone metastases had slightly shrunk (images not shown). Furthermore, the serum ALP and LDH had decreased to normal levels (Fig. 1I); therefore, continuous systemic treatment was scheduled.

However, 26 days after the initiation of the 4th cycle of chemotherapy, the patient was admitted for a thorough examination due to rapidly worsening nausea and lower limb weakness. Spinal and brain magnetic resonance (MR) imaging with gadolinium revealed diffuse enhancement on the surface of spinal cord, cerebrum and brainstem. In particular, the cerebellar fissures were markedly enhanced (Fig. 1G). On fluid-attenuated inversion recovery images, diffuse abnormal signal intensity was observed in the subarachnoid space (Fig. 1H). These results, even without examination of the cerebrospinal fluid, were sufficient to diagnose MC. Treatment, including whole-brain radiotherapy for $\mathrm{MC}$, was scheduled; however, the patient's condition rapidly deteriorated and he succumbed to the disease 14 days after the diagnosis of MC. 


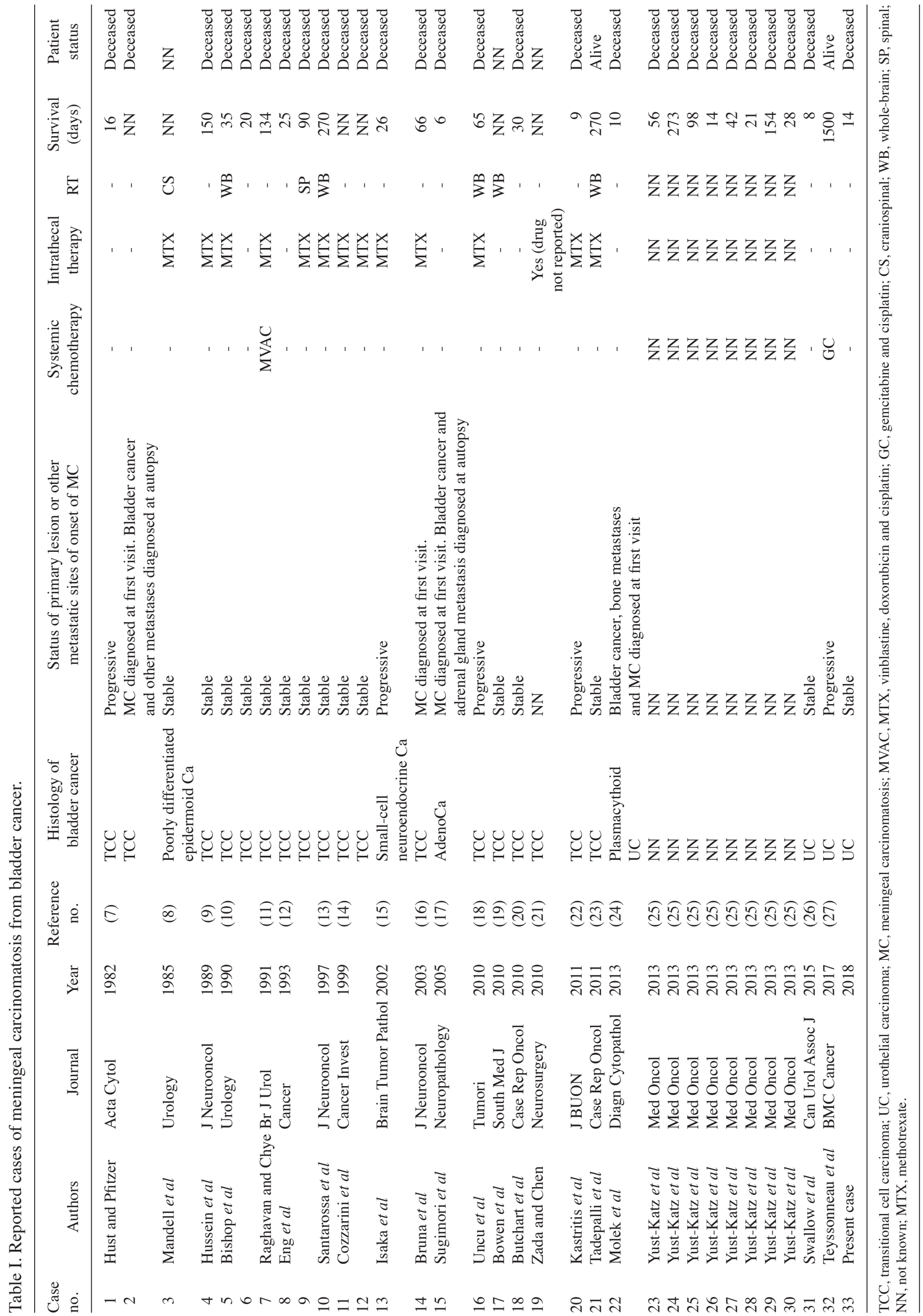


An autopsy was performed to determine the cause of his rapid deterioration and death following the initial diagnosis of MC. The autopsy revealed that tumor cells, which were similar to the bladder cancer cells observed following cystectomy, diffusely infiltrated the subarachnoid space of the cerebrum, cerebellum, brainstem and spinal cord (Fig. 1C and D). Mediastinal and lung hilar lymph node metastases, as well as multiple bone metastases, were also found; however, there were no other findings, such as infection, allergy, metabolic disorders, or cardiac disease. Therefore, MC from bladder cancer was definitively diagnosed as the direct cause of death.

\section{Discussion}

To the best of our knowledge, 33 cases of MC from bladder cancer, including the present case, have been reported to date (Table I) (7-27). In the majority of the cases, the histology of bladder cancer was transitional cell or urothelial carcinoma; however, MC from small-cell neuroendocrine carcinoma or adenocarcinoma was also reported $(15,17)$.

MC is diagnosed in $\sim 10 \%$ of patients with metastatic cancer during the clinical course. Among those cases, it occurs in $<10 \%$ at the time of diagnosis, and in $20 \%$ at the time of first progression after initial treatment (1). Among all cases of $\mathrm{MC}$ from bladder cancer, including the present case, 24 cases were described in detail at the onset of MC (Table I). Even in non-muscle-invasive bladder cancer, a case of MC with multiple bone metastases following transurethral resection with intravesical Bacillus Calmette-Guerin instillation was reported (case no. 32, Table I) (27). MC had already developed at initial diagnosis of bladder cancer in 4 cases (16.7\%). Among the 20 cases with MC developing during treatment for bladder cancer, $4(16.7 \%)$ had synchronously progressive primary or other metastatic sites, while $16(66.6 \%)$ did not.

There is no established treatment for MC from solid tumors, including breast cancer, lung cancer and melanoma, as it is such a rare occurrence. Generally, systemic chemotherapy based on the primary tumor, intrathecal therapy and radiotherapy are implemented. The aim in the majority of the cases is to improve or maintain the patient's quality of life, and to prevent or delay neurological deterioration, as notable prolongation of survival is rare (1). Among 24 cases that received treatment for MC from bladder cancer (Table I), systemic chemotherapy, intrathecal therapy and radiotherapy were administered to $2(8.3 \%), 14(58.3 \%$ ) (single-agent methotrexate in 13 and unknown in 1) and 7 (29.2\%) (whole-brain radiotherapy in 5, craniospinal radiotherapy in 1 and spinal radiotherapy in 1) cases, respectively. The remaining 9 cases $(37.5 \%)$ did not receive any treatment for $\mathrm{MC}$ due to rapid disease progression. The prognosis of MC from bladder cancer is very poor [median survival time from the diagnosis of MC, 35 days (interquartile range: 16-134 days)], although there was one case with a survival duration of $\sim 50$ months (case no. 32, Table I). Other cases with relatively long survival were also reported: Case no. 7, treated by systemic chemotherapy [methotrexate, vinblastine, doxorubicin and cisplatin (MVAC)] and intrathecal methotrexate (survival time, 134 days) (11), and case no. 21 , treated with intrathecal methotrexate and whole-brain radiotherapy (survival time: 270 days) (23).
Methotrexate is one of the key drugs for the treatment of $\mathrm{MC}$, along with cytarabine or thiotriethylenephosphoramide, and it is administered as systemic and/or intrathecal chemotherapy (1). In systemic chemotherapy for bladder cancer, methotrexate is widely used in the MVAC regimen as the first-line therapy, as well as in the GC regimen (28). In the present case, although the GC regimen was selected as salvage chemotherapy, the oncological outcome may have been different by reducing a risk of the onset of MC if the MVAC regimen, including methotrexate (which penetrates cerebrospinal fluid relatively easily), had been deployed.

In MC from other cancers, the effectiveness of molecular targeted therapy or immune checkpoint inhibitors, such as bevacizumab combined with etoposide and cisplatin for breast cancer (29), intrathecal trastuzumab for human EGFR-related 2 (HER2) positive breast cancer (30) and nivolumab for lung adenocarcinoma (31), was reported. The efficacy of an immune checkpoint inhibitor in bladder cancer has also been reported (32). Although it is unclear whether these drugs are effective for MC from bladder cancer, a promising strategy for MC may be expected to be designed in the future.

MC from solid tumors, including bladder cancer, may become more common, as cancer patients survive longer due to the more effective treatments (25). In the present case, the MR imaging findings matched the pathological findings on autopsy. Cerebrospinal imaging is not a practice routinely recommended for bladder cancer; however, it is necessary to perform this as soon as possible when the patient has symptoms that suggest meningitis, even if the primary lesion or other metastatic sites remain stable during systemic treatment.

\section{Acknowledgements}

The authors would like to thank the pathologist Dr Mika Sakaki (Department of Diagnostic Pathology, Saitama Medical University International Medical Center) for her valuable support in the autopsy.

\section{Funding}

No funding was received.

\section{Availability of data and materials}

Not applicable.

\section{Ethics approval and consent to participate}

Not applicable.

\section{Patient consent for publication}

Opt-out consent was applied in this case report.

\section{Authors' contributions}

YU, SS and GK planned the case study, and collected the data and the images of the case in addition to producing the draft of the manuscript. KN and MO for urology, YO and AU 
for diagnostic radiology, and MY for diagnostic pathology critically revised this manuscript for important intellectual content. All authors read and approved the final manuscript to be published.

\section{Competing interests}

All authors declare that they have no competing interests to disclose.

\section{References}

1. Le Rhun E, Weller M, Brandsma D, Van den Bent M, de Azambuja E, Henriksson R, Boulanger T, Peters S, Watts C, Wick W, et al; EANO Executive Board and ESMO Guidelines Committee: EANO-ESMO Clinical Practice Guidelines for diagnosis, treatment and follow-up of patients with leptomeningeal metastasis from solid tumours. Ann Oncol 28 (Suppl 4): iv84-iv99, 2017.

2. Chamberlain MC: Leptomeningeal metastasis. Curr Opin Oncol 22: 627-635, 2010

3. Roth P and Weller M: Management of neoplastic meningitis. Chin Clin Oncol 4: 26, 2015

4. Le Rhun E, Taillibert S and Chamberlain MC: Carcinomatous meningitis: Leptomeningeal metastases in solid tumors. Surg Neurol Int 4 (Suppl 4): S265-S288, 2013.

5. Gleissner B and Chamberlain MC: Neoplastic meningitis. Lancet Neurol 5: 443-452, 2006.

6. van der Ree TC, Dippel DW, Avezaat CJ, Sillevis Smitt PA, Vecht CJ and van den Bent MJ: Leptomeningeal metastasis after surgical resection of brain metastases. J Neurol Neurosurg Psychiatry 66: 225-227, 1999.

7. Hust MH and Pfitzer P: Cerebrospinal fluid and metastasis of transitional cell carcinoma of the bladder. Acta Cytol 26: 217-223, 1982.

8. Mandell S, Wernz J, Morales P, Weinberg H and Steinfeld A: Carcinomatous meningitis from transitional cell carcinoma of bladder. Urology 25: 520-521, 1985.

9. Hussein AM, Savaraj N, Feun LG, Ganjei P and Donnelly E: Carcinomatous meningitis from transitional cell carcinoma of the bladder: Case report. J Neurooncol 7: 255-260, 1989.

10. Bishop JR Jr, Moul JW, Maldonado L and McLeod DG: Transitional cell carcinomatous meningitis after M-VAC (methotrexate, vinblastine, doxorubicin, and cisplatin) chemotherapy. Urology 36: 373-377, 1990.

11. Raghavan D and Chye RW: Treatment of carcinomatous meningitis from transitional cell carcinoma of the bladder. Br J Urol 67: 438-440, 1991.

12. Eng C, Cunningham D, Quade BJ, Schwamm L, Kantoff PW and Skarin AT: Meningeal carcinomatosis from transitional cell carcinoma of the bladder. Cancer 72: 553-557, 1993.

13. Santarossa S, Vaccher E, Balestreri L, Volpe R and Tirelli U: Solitary meningeal recurrence in a patient with transitional cell carcinoma of the bladder with locally bulky disease at presentation. J Neurooncol 35: 141-143, 1997.

14. Cozzarini C, Reni M, Mangili F, Baldoli MC, Galli L and Bolognesi A: Meningeal carcinomatosis from transitional cell carcinoma of the bladder: Report of two cases and review of the literature. Cancer Invest 17: 402-407, 1999.

15. Isaka T, Maruno M, Sato M, Kinoshita M, Nishida T, Kiyohara $H$ and Yoshimine T: Brain metastasis from small-cell neuroendocrine carcinoma of the urinary bladder: A case report. Brain Tumor Pathol 19: 117-122, 2002.
16. Bruna J, Rojas-Marcos I, Martínez-Yelamos S, Català I, Vidaller A, Galán C, Krupinski J and Rubio F: Meningeal carcinomatosis as the first manifestation of a transitional cell carcinoma of the bladder. J Neurooncol 63: 63-67, 2003.

17. Sugimori K, Kobayashi K, Hayashi M, Sakai N, Sasaki M and Koshino Y: Leptomeningeal carcinomatosis from urinary bladder adenocarcinoma: A clinicopathological case study. Neuropathology 25: 89-94, 2005.

18. Uncu D, Arpaci F, Beyzadeoglu M, Gunal A, Surenkok S, Ozturk M and Ozet A: Meningeal carcinomatosis: An extremely rare involvement of urinary bladder carcinoma. Tumori 96: 352-354, 2010.

19. Bowen CD, Von Burton G, Bargen RC, Madonia P, Zhang S, Toledo EG, Zweig R and Pant C: Carcinomatous meningitis secondary to transitional cell bladder cancer. South Med J 103: 809-812, 2010.

20. Butchart C, Dahle-Smith A, Bissett D, Mackenzie JM and Williams DJ: Isolated Meningeal Recurrence of Transitional Cell Carcinoma of the Bladder. Case Rep Oncol 3: 171-175, 2010.

21. Zada G and Chen TC: A novel method for administering intrathecal chemotherapy in patients with leptomeningeal metastases and shunted hydrocephalus: case report. Neurosurgery 67 : onsE306-307, 2010.

22. Kastritis E, Zagouri F, Dimopoulos MA and Papadimitriou CA: Carcinomatous meningitis from transitional cell carcinoma of the urinary bladder. J BUON 16: 373-374, 2011.

23. Tadepalli S, Coleman T, Hacket LA and Liles GB: Carcinomatous meningitis: The natural history of successfully treated metastatic bladder cancer. Case Rep Oncol 4: 406-412, 2011.

24. Molek KR, Seili-Bekafigo I, Štemberger C, Jonjić N, Đordević G and Duletić-Načinović A: Plasmacytoid urothelial carcinoma diagnostic challenge in cytology. Diagn Cytopathol 41: 369-373, 2013.

25. Yust-Katz S, Mathis S and Groves MD: Leptomeningeal metastases from genitourinary cancer: The University of Texas MD Anderson Cancer Center experience. Med Oncol 30: 429, 2013.

26. Swallow TW, Mabbutt S and Bell CR: Muscle invasive bladder cancer culminating with leptomeningeal carcinomatosis. Can Urol Assoc J 9: E903-E904, 2015.

27. Teyssonneau D, Daste A, Dousset V, Hoepffner JL, Ravaud A and Gross-Goupil M: Metastatic non-muscle invasive bladder cancer with meningeal carcinomatosis: Case report of an unexpected response. BMC Cancer 17: 323, 2017.

28. Alfred Witjes J, Lebret T, Compérat EM, Cowan NC, De Santis M, Bruins HM, Hernández V, Espinós EL, Dunn J, Rouanne M, et al: Updated 2016 EAU Guidelines on Muscle-invasive and Metastatic Bladder Cancer. Eur Urol 71: 462-475, 2017.

29. Wu PF, Lin CH, Kuo CH, Chen WW, Yeh DC, Liao HW, Huang SM, Cheng AL and Lu YS: A pilot study of bevacizumab combined with etoposide and cisplatin in breast cancer patients with leptomeningeal carcinomatosis. BMC Cancer 15: 299, 2015.

30. Zagouri F, Sergentanis TN, Bartsch R, Berghoff AS, Chrysikos D, de Azambuja E, Dimopoulos MA and Preusser M: Intrathecal administration of trastuzumab for the treatment of meningeal carcinomatosis in HER2-positive metastatic breast cancer: A systematic review and pooled analysis. Breast Cancer Res Treat 139: 13-22, 2013.

31. Gion M, Remon J, Caramella C, Soria JC and Besse B: Symptomatic leptomeningeal metastasis improvement with nivolumab in advanced non-small cell lung cancer patient. Lung Cancer 108: 72-74, 2017

32. Massari F, Di Nunno V, Cubelli M, Santoni M, Fiorentino M, Montironi R, Cheng L, Lopez-Beltran A, Battelli N and Ardizzoni A: Immune checkpoint inhibitors for metastatic bladder cancer. Cancer Treat Rev 64: 11-20, 2018. 